Cuad. Soc. Esp. Cienc. For. 46(1): 33-56 (2020)

Doi: https://doi.org/10.31167/csecfv0i46.19902

Sociedad Española

\title{
Seguimiento de plagas y enfermedades forestales en Andalucía: interpretación a diferentes escalas
}

\author{
Pest and disease monitoring of forests in Andalusia: \\ interpretation at different scales
}

\author{
Navarro-Cerrillo, R.M, ${ }^{1 *}$; Ruiz-Gómez, F.J. ${ }^{1}$ \\ ${ }^{1}$ Departamento de Ingeniería Forestal, Universidad de Córdoba. Grupo de Evaluación \\ y Restauración de Sistemas Agrícolas y Forestales (PAIDI-RNM-360). Ed. Leonardo Da Vinci, P1. \\ Campus U. Rabanales. CT N-IV, km 396. 14071 Córdoba. \\ RMNC: rmnavarro@uco.es; FJRG: g72rugof@uco.es
}

*Autor para correspondencia: rmnavarro@uco.es 


\title{
Resumen
}

La vigilancia y el seguimiento de plagas y enfermedades forestales se han convertido en una actividad clave para la conservación, la producción y la gestión de los sistemas forestales. Estos sistemas permiten iniciar rápidamente medidas de contención, control o en su caso erradicación que eviten la propagación y el establecimiento permanente de estos agentes, reduciendo los costos y los daños económicos y ambientales. En Andalucía, existen diferentes niveles de información sobre sanidad forestal: i) el seguimiento del estado de los bosques mediante Redes sistemáticas de Equilibrios Biológicos (Red-SEDA), que se dirigen a una escala regional y a una detección temprana de alerta; ii) los enfoques locales de vigilancia basado en datos de inventarios; y iii) los censos sistemas de trampeo (trampas con cebo, árboles centinela, etc.). La integración de esta información, y la incorporación de otros sistemas de evaluación como la teledetección y la ciencia ciudadana, permiten crear sistemas complementarios de inspección, seguimiento y control para garantizar la bioseguridad forestal en Andalucía. En esta revisión, se analiza la experiencia existente sobre estos niveles de información, destacando sus fortalezas y debilidades, así como su complementariedad e integración en un sistema regional de sanidad forestal, con el fin de optimizar el uso de cada nivel de información y las opciones más adecuadas para reforzar un programa integral de bioseguridad forestal a partir de la información presentada.

Palabras clave: sanidad forestal, plagas y enfermedades, Redes ICP, inventarios forestales, censos.

\begin{abstract}
Monitoring of forest pests and diseases have become a key activity for the conservation, production and management of forest ecosystems. These monitoring systems make possible to assess, contain, and control forest pest and diseases or, where appropriate, eradication measures that prevent the spread and permanent establishment of these agents, reducing costs and economic and environmental damage. In Andalusia, there are different levels of information on forest health: i) monitoring the state of forests through systematic networks (Red-SEDA), aimed at a regional scale and early warning detection; ii) local approaches based on forest inventories; and iii) censuses. The integration of these information levels, and the incorporation of other assessment systems such as remote sensing, citizen science and wireless ecophysiological networks, allow the creation of complementary monitoring and control systems to guarantee forest biosecurity in Andalusia. In this review, the existing experience on these levels of information is analyzed, highlighting their strengths and weaknesses, as well as their complementarity and integration in a regional forest health system to optimize the use of each information level and reinforcing a comprehensive forest biosecurity program.
\end{abstract}

Keywords: forest health, pests and diseases, ICP Networks, forest inventories, censuses, 


\section{Introducción}

Los insectos y los microorganismos son una parte muy importante de los ecosistemas naturales, siendo en ocasiones considerados como un factor regulador de los ecosistemas forestales. Sin embargo, en algunos casos la dinámica de ciertas especies de insectos, hongos, y otros microorganismos pueden causar daños muy importantes en masas forestales, tanto en ecosistemas naturales, como seminaturales o en masas artificiales. Estos daños provocan cambios bruscos en la estructura y función de los ecosistemas forestales, que se traducen por ejemplo en la pérdida de crecimiento o en el desencadenamiento de procesos de mortalidad de distinta gravedad (Ayres y Lombardero, 2017). El nivel y gravedad de los daños causados dependen, entre otros, de la virulencia del agente, la severidad de los daños y su capacidad para extenderse, produciendo perturbaciones que podrían ser localizadas, afectando a escala de árbol, hasta cubrir extensiones de cientos o miles de hectáreas. Las perturbaciones bióticas, por tanto, suponen una reducción temporal o permanentemente del equilibrio en un ecosistema forestal, dando lugar a una reducción o a la pérdida total de su capacidad biológica de crecimiento, reproducción, y supervivencia, lo que se traduce en la perdida de producciones y servicios de los bosques (Boyd et al., 2013). El cambio climático ha agravado muchos de estos procesos a diferentes escalas, aumentando la frecuencia, severidad y duración de las plagas y enfermedades forestales (Loehle et al., 2016). Estos posibles impactos son particularmente relevantes para los bosques Mediterráneos, donde las perturbaciones relacionadas con los incendios y la sanidad forestal presentan una gran influencia en la dinámica del ecosistema. El impacto de los procesos de decaimiento forestal resulta cada vez más evidente en todo el mundo (Trumbore et al., 2015), y especialmente en Andalucía, donde afecta a numerosos ecosistemas de las principales especies forestales de la región (Navarro-Cerrillo et al., 2014, 2018; Ruiz-Gómez et al., 2019).

El desarrollo de sistemas de evaluación y seguimiento de sanidad forestal se presenta como una herramienta fundamental en el seguimiento de los procesos de decaimiento forestal. La integración de diferentes fuentes de información a escalas temporales y espaciales distintas desempeñarán en estas redes de seguimiento un papel fundamental para monitorear la ocurrencia y evaluar los impactosde estas perturbaciones (McDowell et al., 2015). Estos sistemas deben aportar información sobre la presencia, la extensión, y la severidad de los diferentes agentes implicados, y generar datos útiles para la gestión forestal, pero también para la investigación y modelización de dichos procesos. Los programas de monitoreo de plagas forestales se han llevado a cabo de manera intensiva en España desde hace muchas décadas (e.j. procesionaria del pino Thaumetopoea pityocampa (Denis y Schiffermuller)); sin embargo, la variedad de ecosistemas afectados, de potenciales agentes y de interacción entre los distintos factores implicados, exige cuantificar y comprender estos procesos y su impacto en la dinámica de los ecosistemas forestales, lo que supone un importante desafío metodológico y operativo. La integración de diferentes sistemas de seguimiento a diferentes escalas espaciales y temporales es un requisito previo fundamental para el desarrollo de políticas forestales, y pueden resultar en el desarrollo de nuevos métodos para monitorear, evaluar impactos, elaborar carto- 
grafía y desarrollar técnicas de gestión. Andalucía viene realizando desde hace más de dos décadas el seguimiento de plagas y enfermedades forestales usando diferentes aproximaciones, por lo que representa un buen ejemplo para mostrar las fortalezas y debilidades de este tipo de programas. El objetivo de este trabajo es describir y valorar las capacidades y funciones de cada uno de los niveles de información para la detección, evaluación y control de daños bióticos en sistemas forestales. Este documento se basa, en gran medida, en la experiencia compartida del Departamento de Sanidad Forestal de la Consejería de Agricultura, Pesca y Desarrollo Rural de la Junta de Andalucía, y del Grupo de Investigación de Evaluación y Restauración de Sistemas Agrícolas y Forestales (PAIDI-RNM-360) de la Universidad de Córdoba.

\section{Red Andaluza de Equilibrios Biológicos de Ecosistemas Forestales}

\subsection{La Red SEDA}

E1 Reglamento CEE 3528/86 sobre protección de bosques contra los efectos de la contaminación atmosférica puso en marcha una serie de acciones de seguimiento del estado de los ecosistemas forestales en todos los países comunitarios, que dio lugar a los inventarios nacionales de daños forestales (IDF) (Sánchez Peña et al.,

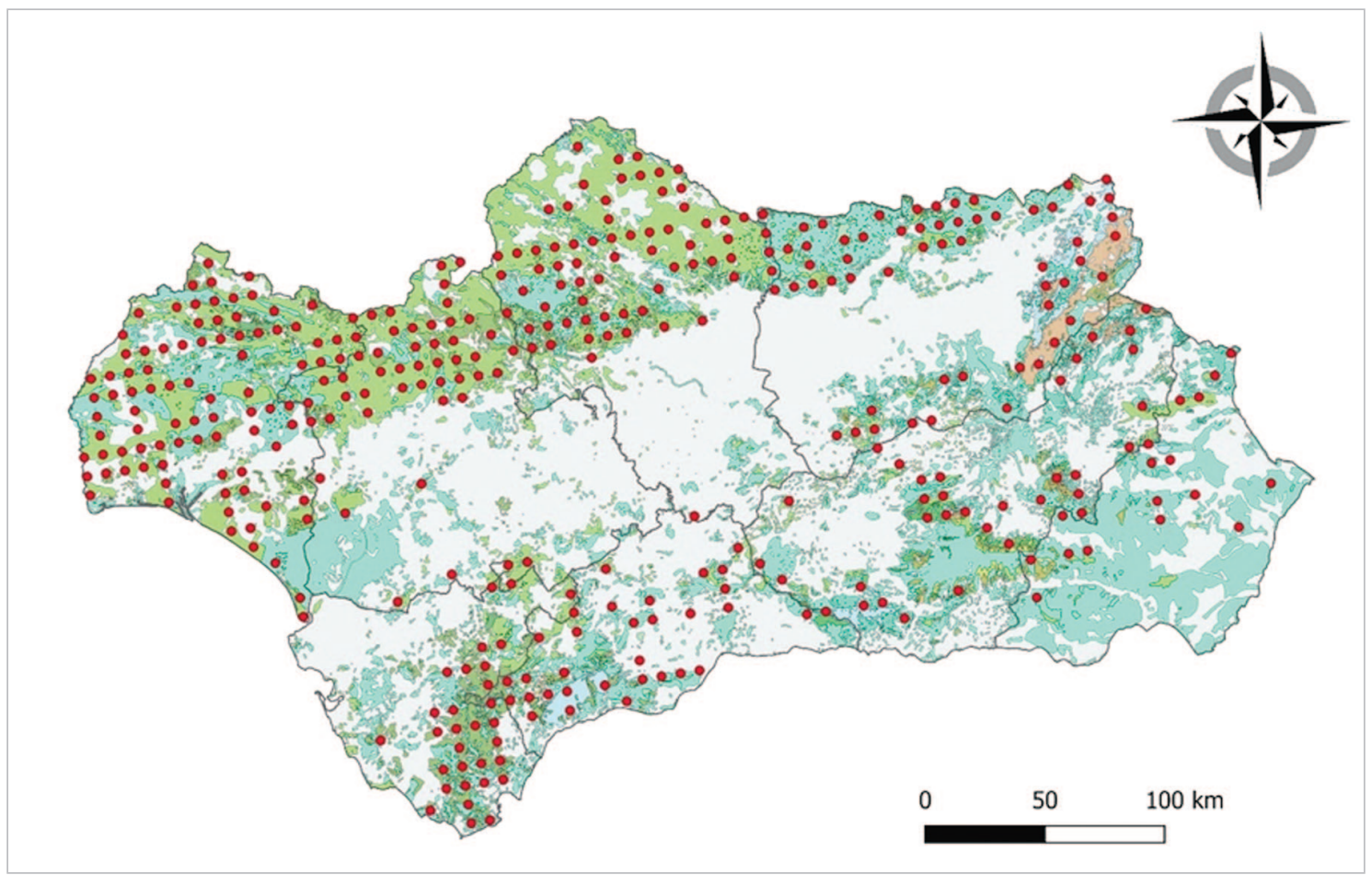

Figura 1. Asignación de los puntos de muestreo de la Red de Daños de Andalucía con una malla de $8 \mathrm{x}$ $8 \mathrm{~km}$ sobre zonas forestales. Las áreas coloreadas en la figura representan los principales tipos forestales recogidos en el Mapa Forestal de España MFE200 (MITECO, 1997). 
2009). Los IDF se corresponden con muestreos sistemáticos, con periodicidad anual, de la evolución del estado de salud de los bosques, que abarcan el total de la superficie forestal comunitaria sobre una malla de 16 x $16 \mathrm{~km}$. (Nivel I) (Reglamento 1696/87; 926/93).

La Junta de Andalucía, a través de la Consejería con competencias en la gestión forestal, lanzó en el año 2000 una Red Autonómica de Equilibrios Biológicos, integrada inicialmente por la Red Andaluza de Seguimiento de Daños sobre Ecosistemas Forestales (Red SEDA), sobre la base de la malla kilométrica existente (16 x 16 $\mathrm{km}$ ), pero con malla densificada de $8 \times 8 \mathrm{~km}$ (Fig. 1). El establecimiento y medición de las parcelas de la Red SEDA se realizó de acuerdo al procedimiento empleado en la Red Europea de Seguimiento de Daños en los Bosques (ICP Forest) (Lorenz, 1995; SSF - DGDRyPF, 2012), y se adaptó para el caso de la Red SEDA (NavarroCerrillo et al, 2000).

La Red SEDA cuenta con 374 puntos, donde se realiza el seguimiento del estado fitosanitario de más de 8800 individuos pertenecientes a 118 especies distintas, considerando especies frondosas y coníferas, de porte arbóreo y matorral. De este modo abarca los principales tipos de ecosistema, y todas las especies forestales principales presentes en Andalucía (Tabla 1), siendo las especies más abundantes la encina, el pino piñonero, el alcornoque y el pino carrasco.

Tabla 1. Número de parcelas de muestreo de la Red Andaluza de Equilibrios Biológicos en ecosistemas forestales según especies forestales principales.Se han considerado las especies con una presencia igual o superior al 50\% en la parcela. Datos de la campaña de 2018 disponibles en www.juntadeandalucia. es/medioambiente/site/rediam

\begin{tabular}{llc}
\hline \multicolumn{1}{c}{ Especie } & \multicolumn{1}{c}{ Nombre comun } & Parcelas \\
Quercus ilex & Encina, carrasca, chaparra & 168 \\
Pinus pinea & Pino piñonero, pino doncel & 46 \\
Quercus suber & Alcornoque & 44 \\
Pinus halepensis & Pino carrasco & 37 \\
Olea europaea & Acebuche & 26 \\
Pinus pinaster & Pino resinero, pino negral & 19 \\
Eucalyptus globulus & Eucalipto blanco & 19 \\
Pinus nigra & Pino negral, pino salgareño & 11 \\
Eucalyptus camaldulensis & Eucalipto rojo & 8 \\
Pinus sylvestris & Pino albar, pino de Balsaín, pino silvestre & 4 \\
Quercus canariensis & Quejigo, roble & 2 \\
Castanea sativa & Castaño & 2 \\
Phillyrea latifolia & Agracejo, labiérnago prieto & 1 \\
Quercus faginea & Quejigo, roble & 1 \\
Fraxinus angustifolia & Fresno & 1 \\
Populusnigra, Populus x canadensis & Chopo & 1 \\
Arbutusunedo, Arbutus canariensis & Madroño, madroñero & 1 \\
Prunus sp. & Espino negro, endrino, cerezo silvestre & 1 \\
Pistacia lentiscus & Charneca, lentisco & 1 \\
\hline
\end{tabular}




\subsection{La aplicación de la Red SEDA al seguimiento de los procesos de decaimiento}

La Red SEDA ha permitido crear una base territorial para el seguimiento de agentes bióticos y abióticos, y su evolución temporal, (2001-2019). La información recogida en los puntos de la Red, asociados a análisis específicos de determinados agentes bióticos está ayudando a una mejor comprensión de los procesos de dispersión de agentes bióticos de gran importancia para la sanidad de los ecosistemas forestales (Figura 2).Además, la Red SEDA ha permitido identificar plagas emergentes relacionadas con problemas de sanidad forestal en Andalucía (Matsucoccus

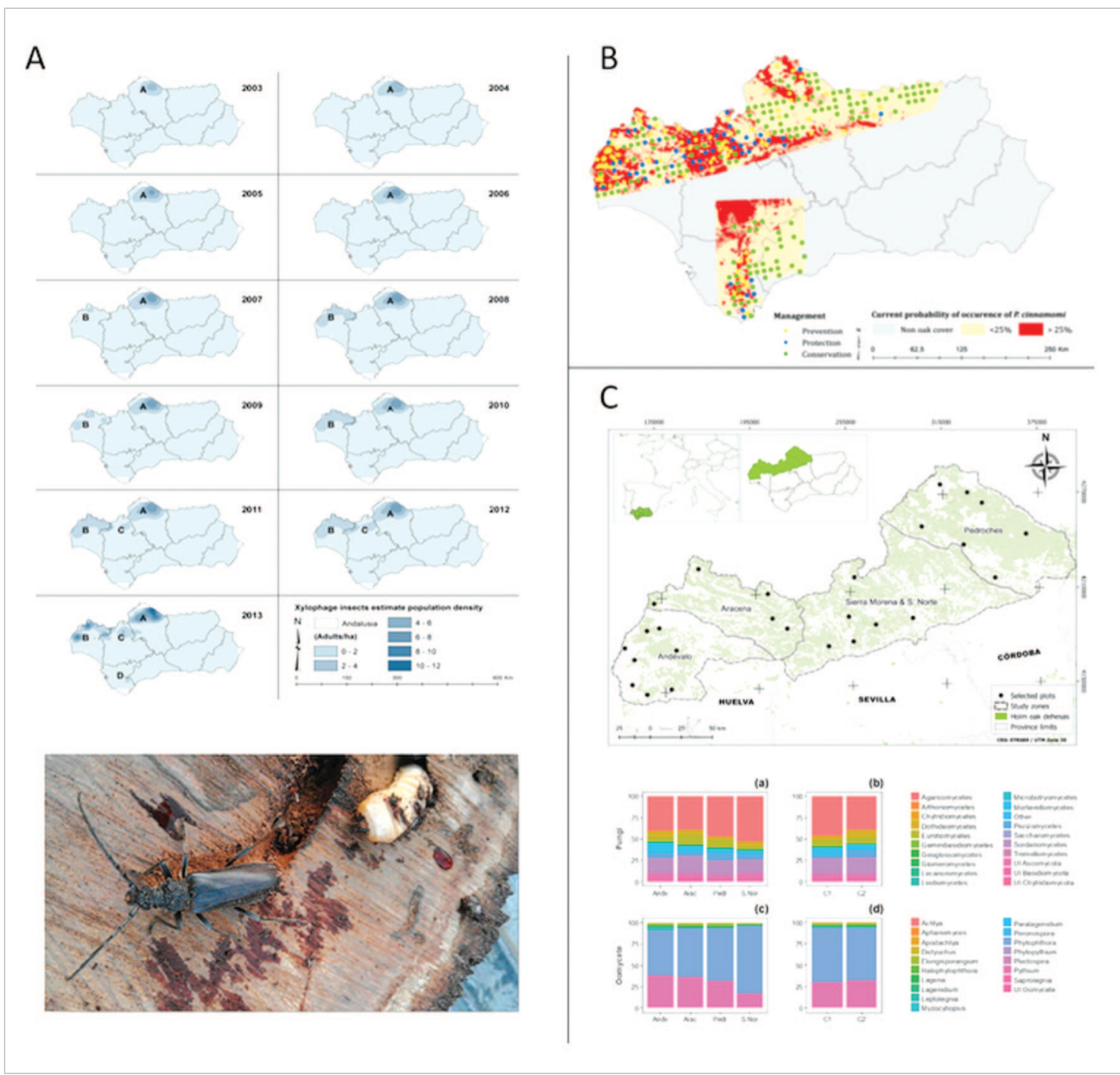

Figura 2. Algunos ejemplos de análisis de presencia y evolución de agentes bióticos a partir de datos de la Red de Equilibrios Biológicos. A) Evaluación de presencia de Cerambyx en Andalucía (Duque-Lazo y Navarro Cerrillo, 2017). B) Evaluación del área de dispersión potencial de P. cinnamomi en Andalucía (Duque-Lazo et al., 2018). C) Evaluación de la relación entre biodiversidad microbiana y el decaimiento de encina (Ruiz Gómez et al., 2019). 
feytaudi Ducasse, 1941-cochinilla del pino rodeno, o Candidatus Phytoplasma pini en masas de pino carrasco), así como para el diagnóstico rápido y exacto de nuevos agentes nematodos de madera de pino (Bursaphelenchus xylophilus (Steiner et Buhrer)).

Los diferentes trabajos científicos derivados a partir de los datos de la Red SEDA ponen en evidencia la importancia de este tipo de sistemas de seguimiento de cambios a nivel regional, aportando información muy valiosa respecto a aspectos tales como los patrones de distribución espacial y temporal de agentes (Duque-Lazo et al., 2016; Duque-Lazo y Navarro-Cerrillo, 2017), el desarrollo de estrategias de gestión (Duque-Lazo et al., 2018; (Duque-Lazo et al., 2018), relación entre los factores ambientales y enfermedades forestales (Ruiz Gómez et al., 2019), o la dinámica selvicola de masas forestales afectadas por decaimiento (Sánchez-Salguero et al., 2013; Rodriguez-Vallejo y Navarro-Cerrillo, 2019), y que son esenciales para la toma de decisiones sobre estrategias de gestión forestal.

\subsection{Ventajas e inconvenientes de las Redes sistemáticas de seguimiento de bosques}

Las redes de Daños sobre Ecosistemas Forestales tienen las siguientes ventajas:

* Establecen un procedimiento metodológico normalizado en el tiempo y en el espacio para la obtención de información sobre sanidad forestal (autonómico, nacional y europeo). La utilización de mismos protocolos de trabajo garantiza el intercambio de información (nivel nacional e interregional) entre zonas ecológicas con problemas análogos.

* Permiten obtener información técnica y científica sobre el estado fitosanitario de los ecosistemas forestales, que orienta a la hora de definir políticas para conservar y mejorar los ecosistemas forestales.

* Incorporan nuevos programas de seguimiento de agentes que se puedan proponer desde otros ámbitos y, por tanto, permiten incorporar y actualizar el uso de nuevas tecnologías de seguimiento, aumentando la complejidad de los programas.

* La Red de Nivel I es también una malla de información extremadamente versátil para estudios de ecología forestal, en la que la toma de datos se hace sobre una misma base territorial, lo que permite aumentar el valor de la información, creando un sistema específico de seguimiento de ecosistemas forestales.

* El mantenimiento de la Red en el tiempo refuerza las potencialidades y permite corregir los puntos débiles de la misma. La relevancia y el valor potencial de los resultados obtenidos y el interés múltiple de mantener esta acción común garantizan, en nuestra opinión, la continuidad de los trabajos. 
Sin embargo, las Redes presentan limitaciones para la evaluación de procesos relacionados con la sanidad forestal:

* Las plagas y enfermedades presentan patrones de distribución al azar, sobre sistemas forestales que tienen una distribución espacial discontinua, lo que dificulta que los puntos de una red sistemática coincidan con áreas afectadas por este tipo de procesos.

* El establecimiento de Redes es un proceso complejo en tiempo, recursos y formación de técnicos, lo que hacer que sólo empiecen a generar información fiable después de un periodo relativamente largo (entre 5-10 años).

En general, debe aceptarse que las Redes no están pensadas ni diseñadas para el seguimiento de plagas o enfermedades específicas, en particular cuando estos no responden a causas de carácter general que afecten a grandes superficies forestales.

\subsection{Otras aplicaciones de la información procedente de Redes de Seguimiento}

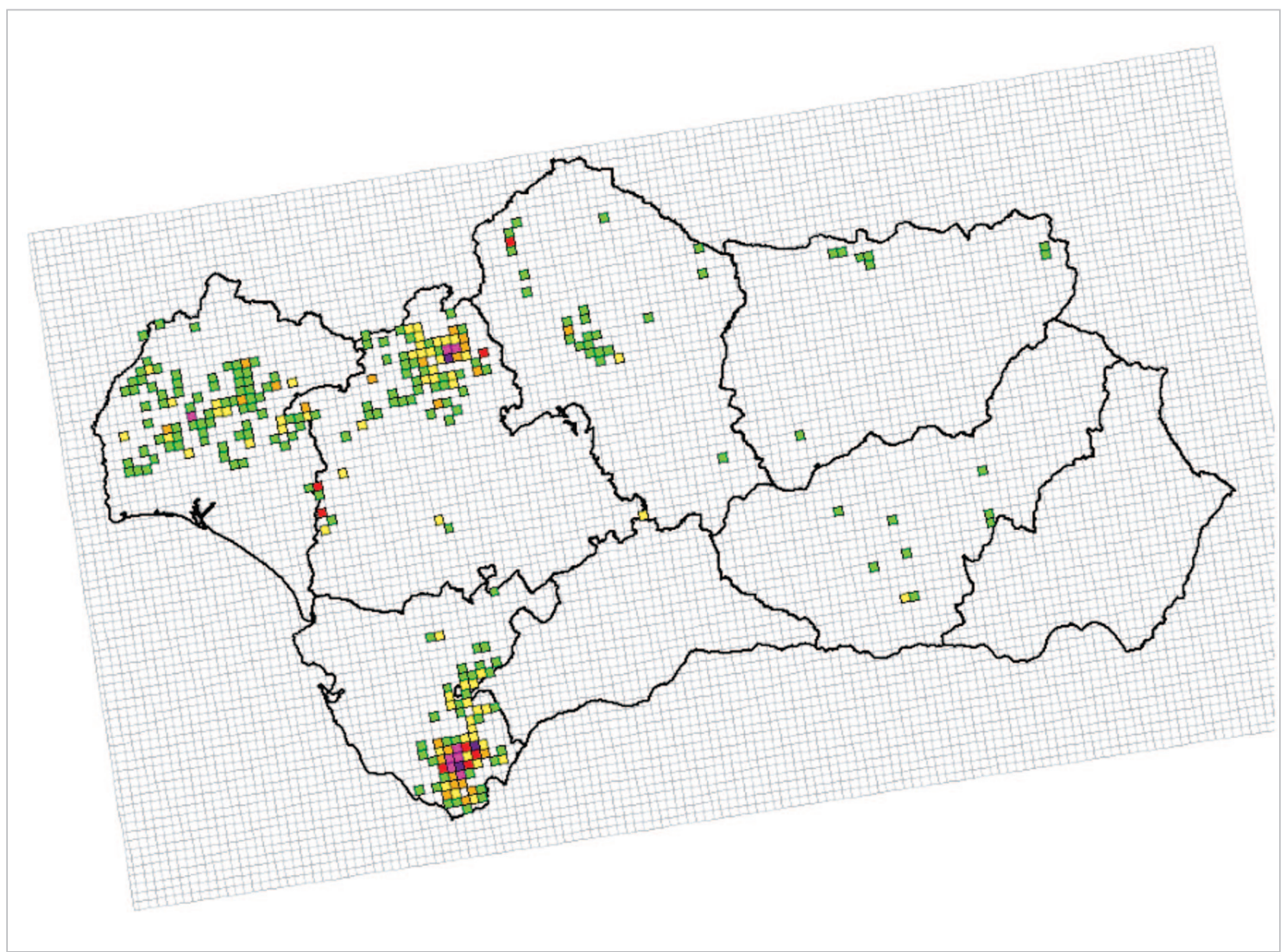

Figura 3. Distribución de los focos de seca sobre masas de Quercus sp. en Andalucía (Navarro Cerrillo et al., 2001). 


\subsubsection{Integración de daños procedentes de censos regionales}

La distribución sistemática de las Redes de Equilibrios Biológicos establece una "estructura territorial" sobre la cual es relativamente sencillo volcar datos procedentes de otras fuentes de información. Por ejemplo, inventarios de presenciadaños a escala regional, que se pueden interpretar como catálogos de daños, adaptados a diferentes agentes y escalas. Un caso particular fue el censo de focos de seca realizados por la Junta de Andalucía en 1999 a escala 1:250.000, que entre sus diferentes objetivos, tenía el analizar. las características ecológicas en las cuales aparecen los focos de seca, y establecer los agentes bióticos más relevantes en el proceso de seca en Andalucía (Navarro Cerrillo et al., 2001) (Figura 3).

Los análisis derivados de la información obtenida en el censo permitieron describir la distribución espacial, las características de los focos (superficie, vegetación, etc.), así como los agentes asociados y la severidad de los daños. Se identificaron muchos agentes que parecían tener una implicación activa en el fenómeno de la seca, y que después han demostrado ser importantes agentes de daño en estos sistemas forestales (Phytophthora cinnamomi Rands; Botryosphaeria stevensii Shoemaker, Diplodia mutila Fries apud Munt, Lymantria dispar L., Tortrix viridana L., grupo Cerambyx (sensu Soria); Prinobius germari Germar).

\section{Evaluación de agentes bióticos en comarcas forestales a partir de inventarios}

\subsection{Inventarios forestales y evaluación de agentes bióticos y abióticos}

Los inventarios forestales permiten el acceso a información muy variada sobre diferentes aspectos (selvícolas, estructurales, biodiversidad, etc.) de los bosques. A nivel estatal y regional existe una inmensa base de datos procedentes de inventarios forestales (Ruiz-Benito y García-Valdés, 2016).Desde su inicio, los datos de los inventarios se han utilizado cada vez más en otros ámbitos distintos a los de la valoración dendrométrica y dasométrica de los montes. Algunas de estas aplicaciones son particularmente significativas para el seguimiento de la sanidad forestal: evaluación de daños, evaluaciones de enfermedades, procesos de cambios de especie asociados al cambio climático, o estudios de la biodiversidad. Los datos procedentes de inventarios tienen muchas ventajas, como son una cierta similitud de los formatos y del contenido, la temporalidad (10 años en términos generales), y la diversidad de situaciones ecológicas y selvícolas que abarcan. Todo ello hace que la cantidad y calidad de la información sea muy importante, y que el procesado de datos sea (relativamente) sencillo.

Como ejemplo del uso de los datos de inventario en una zona particular para la evaluación de daños en masas forestales, se muestra la información disponible para el Parque Natural de Sierra de las Nieves (Figura 4, Tabla 2). En el marco de las colaboraciones con el Programa de restauración de los pinsapares se están estudiando los procesos de regeneración a partir de series temporales de datos de inventario y su implicación con el impacto de agentes bióticos y el cambio climático. 


\subsection{Ventajas e inconvenientes de los inventarios forestales para los estudios de sanidad forestal}

El uso de inventarios forestales para la evaluación de procesos relacionados con la sanidad forestal tiene las siguientes ventajas:

* Permiten obtener información sobre el estado fitosanitario a escala de comarca forestal o monte, unidades normalmente homogéneas desde el punto de vista de gestión, lo que supone un paso previo imprescindible para cualquier plan de control integrado de daños.

* Utilizan procedimientos de inventario normalizados para todos los inventarios, lo que hace comparable (parcialmente) la información, y evita el uso de información inadecuada o subjetiva.

* Incorpora los resultados de los inventarios dentro de los Proyectos y Planes Técnicos de Ordenación, que normalmente son documentos que corresponden a una unidad de gestión forestal.

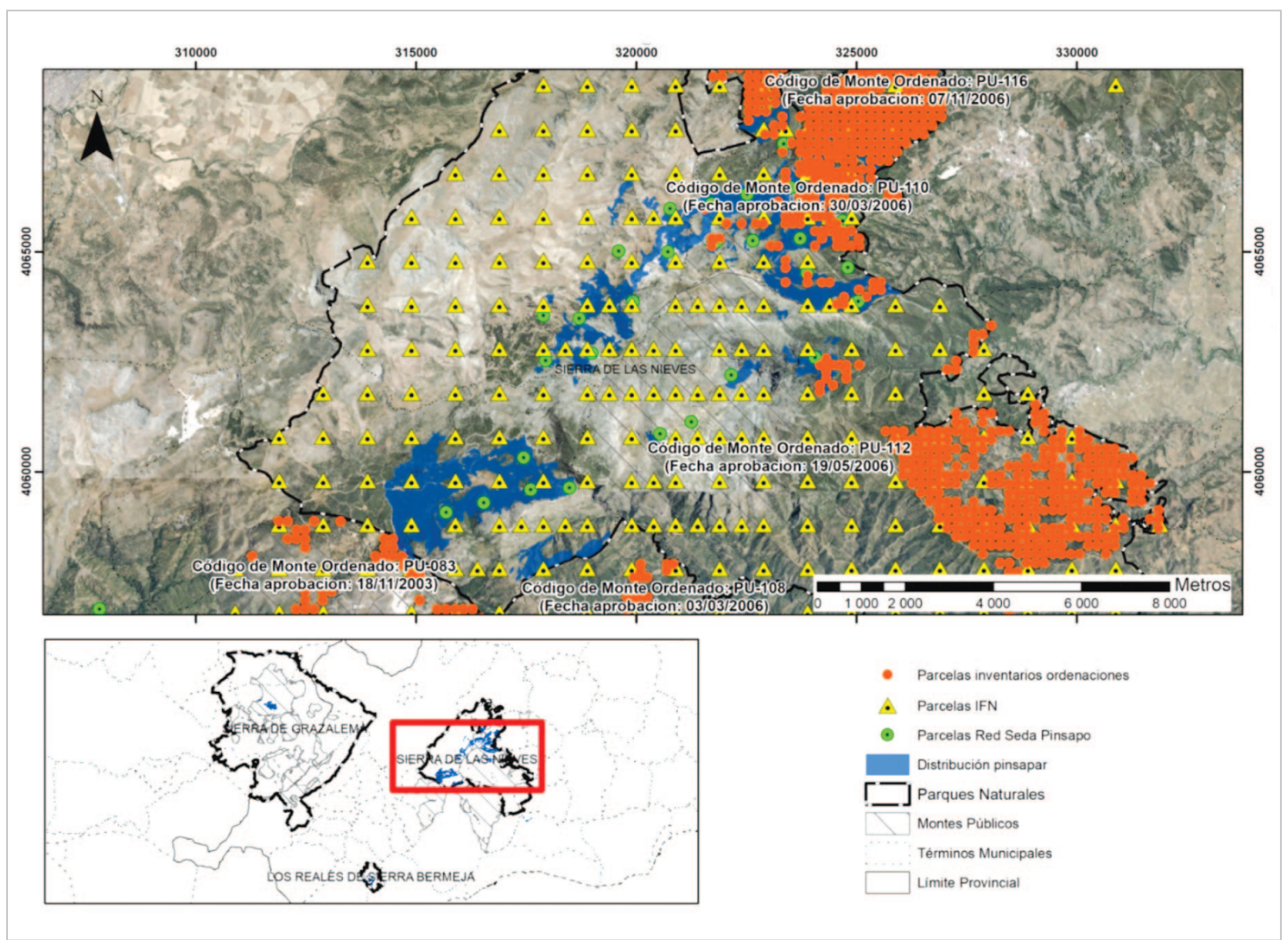

Figura 4. Superposición de las diferentes redes de vigilancia, parcelas y censos utilizados en el estudio de la afección del pinsapar de Sierra de las Nieves. 
Tabla 2. Descripción de las principales características de los inventarios disponibles en los Parques Naturales del área de distribución de los pinsapares (Abies pinsapo Boiss.) en Andalucía en el año 2007.

(Fuente: REDIAM)

\begin{tabular}{|c|c|c|c|}
\hline Monte & Fecha & Parcelas de muestreo & $N^{0}$ de parcelas \\
\hline IFN-3 & & $\begin{array}{l}\text { Malla sobre el Mapa Forestal de España } \\
\text { UTM1-kmx1-km.Parcelas permanentes. } \\
\text { Repetición de parcelas. } \\
\text { Parcelas remedidas aprox. } 85 \% \text {. }\end{array}$ & 181 \\
\hline Red SEDA/PINSAPO & & $\begin{array}{l}\text { Inventario del estado fitosanitario } \\
\text { de la vegetación forestal con muestreo } \\
\text { sistemático con malla de } 1 \mathrm{x} 1 \mathrm{~km}\end{array}$ & 31 \\
\hline $\begin{array}{l}\text { Ordenación Montes públicos del PN } \\
\text { Sierra de Grazalema (PU-040) }\end{array}$ & 2000 & $\begin{array}{l}\text { Inventario sistemático estratificado } \\
\text { con parcelas circulares de radio } 13 \mathrm{~m}\end{array}$ & 1.690 \\
\hline $\begin{array}{l}\text { Ordenación del grupo de montes } \\
\text { "El Burgo" (PU-082) }\end{array}$ & 2003 & $\begin{array}{l}\text { Inventario sistemático estratificado } \\
\text { con parcelas circulares de radio } 13 \mathrm{~m}\end{array}$ & 1.027 \\
\hline $\begin{array}{l}\text { Ordenación monte Sierra Bermeja } \\
\text { (PU-107) }\end{array}$ & 2006 & $\begin{array}{l}\text { Inventario sistemático con parcelas } \\
\text { circulares de radio variable hasta } \\
\text { medición de } 15 \text { pies mayores } \\
\text { pies mayores }\end{array}$ & 604 \\
\hline $\begin{array}{l}\text { Ordenación monte } \\
\text { Pinar de Yunquera (PU-110) }\end{array}$ & 2006 & $\begin{array}{l}\text { Inventario sistemático estratificado con } \\
\text { parcelas circulares de radio } 13 \mathrm{~m}\end{array}$ & 459 \\
\hline $\begin{array}{l}\text { Ordenación } \\
\text { Montes de Tolox (PU-112) }\end{array}$ & 2006 & $\begin{array}{l}\text { Inventario sistemático estratificado con } \\
\text { parcelas circulares de radio de } 18 \mathrm{~m} \text { en } \\
\text { pinar y de } 13 \mathrm{~m} \text { en pinsapar }\end{array}$ & 503 \\
\hline $\begin{array}{l}\text { Ordenación monte } \\
\text { Morenas de Briñuelas (PU-116) }\end{array}$ & 2006 & $\begin{array}{l}\text { Inventario sistemático con parcelas } \\
\text { circulares de radio } 13 \mathrm{~m}\end{array}$ & 71 \\
\hline $\begin{array}{l}\text { Ordenación Montes patrimoniales en } \\
\text { el PN Sierra de Grazalema (PU-275) }\end{array}$ & 2015 & $\begin{array}{l}\text { Inventario sistemático con parcelas } \\
\text { circulares de radio } 14 \mathrm{~m}\end{array}$ & 1.388 \\
\hline
\end{tabular}

* La información relativamente fácil de actualizar si se mantienen las revisiones de los proyectos y planes técnicos de gestión, o de la temporalidad del inventario (IFN).

\section{Censos de agentes bióticos en ecosistemas forestales}

Además de las tendencias espaciales y temporales a escala regional, la variación local de plagas y enfermedades forestales es muy importante en muchos sistemas forestales. El estudio de estos patrones locales se realiza comúnmente mediante el desarrollo de censos de agentes, que permiten comprender su variación espacial-geográfica y la relación con factores involucrados en su establecimiento y expansión (Leather, 2005). Los estudios basados en censos han sido los más frecuentes en la evaluación de plagas/enfermedades forestales, y se han aplicado en numerosas especies de insectos y patógenos forestales (Muñoz López, 2007). 
Un ejemplo de uso de un censo como soporte para la evaluación de la incidencia de una enfermedad forestal es el caso del decaimiento de Abies pinsapo Boiss. por la incidencia del hongo Heterobasidion annosum (Fr.) Bref., (H. abietinum Niemela \& Korhonen) en Andalucía. Este hongo se ha identificado como agente patógeno en formaciones de pinsapo, causando el deterioro y la mortalidad de individuos aislados o parcelas de diferente extensión (Cobos Suárez, 1994; Sánchez et al., 2007; López Quintanilla, 2013). Sus efectos principales son la podredumbre de raíz y tronco, llegando a provocar la muerte de los pies afectados(Smith et al., 1992). Los síntomas aéreos de la enfermedad son genéricos y carecen de valor de diagnóstico, ya que otros patógenos pueden producir síntomas similares: clorosis, marchitez, muerte de árboles aislados o en grupos y pies abatidos por el viento que muestran las raíces podridas(Sinclair y Lyon, 2005). Se trata, por tanto, de un hongo cuya detección puede ser difícil en campo, aunque su identificación sea establecida con facilidad en laboratorio, siendo la observación de los basidiocarpos el criterio más seguro de diagnóstico en el terreno.

En el marco de los daños de $H$. annosum observado en los pinsapares de Andalucía, se procedió a censo específico para la evaluación de los daños que ocasiona, la localización concreta de sus focos o la estimación de su expansión. La metodología para la elaboración de este censo de focos de $H$. annosum se basó en el protocolo de trabajo de la Red Andaluza de Equilibrios Biológicos (Navarro-Cerrillo et $a l ., 2000)$ y la Red de Equilibrios Biológicos en ecosistemas con presencia de Abies pinsapo (1 km x $1 \mathrm{~km}$ ) (Navarro-Cerrillo y Calzado Martínez, 2001). Esta Red está restringida geográficamente a Sierra de las Nieves, Sierra de Grazalema y Los Reales de Sierra Bermeja.

El censo de focos se planteó con un diseño estratificado (limitado al estrato pinsapar) y dirigido, buscando todos los enclaves donde la apariencia del dosel arbóreo o el conocimiento directo o reportado de determinados daños en la masa hicieran sospechar la presencia del patógeno. Dada la dificultad de detección "de visu" de la enfermedad, se consideraron como focos de $H$. annosum aquellas zonas donde se apreciaron pies descalzados con raíces afectadas por la descomposición característica atribuible al patógeno, especialmente cuando se pudo ratificar el diagnóstico por la presencia de basidiocarpos. A su vez, se distinguieron dos tipos de focos según la aparente actividad del patógeno en los mismos: focos antiguos y focos activos. Focos activos fueron aquellos donde pudo establecerse la proximidad temporal del derribo (acícula aún verde en la copa del pie caído, podredumbre fresca en raíces, micelio fresco del hongo creciendo bajo la corteza), y focos antiguos aquellos donde se previó la implicación del patógeno (pies derribados con evidente podredumbre de raíz). De forma adicional, ante la inespecificidad de los síntomas aéreos observados, aquellas zonas visitadas, con síntomas evidentes, pero donde no fuera posible la identificación de $\mathrm{H}$. annosum, fueron incluidas en el censo como áreas de posible infección.

Los resultados más importantes de ese censo pueden consultarse en (Navarro Cerrillo et al., 2003). En particular, este censo permitió identificar los focos de la enfermedad (81 focos, de los que 26 fueron considerados focos activos y 55 focos antiguos), el tipo y localización de las masas más afectadas (pinsapares regulares, 
puros, adultos y muy densos de Sierra de las Nieves), y la aparente vinculación entre el binomio Cryphalus numidicus Eichhoffy H. annosum, siendo común la coincidencia de núcleos de alto nivel de daño por el perforador en focos del patógeno (Figura 5).

\subsection{Ventajas e inconvenientes de los censos para los estudios de sanidad forestal}

Los censos presentan un conjunto de ventajas e inconvenientes en los estudios de sanidad forestal. Las principales ventajas son:

* Ofrecen información del conjunto del territorio con presencia del agente, con posibilidad de georeferenciación de las áreas afectadas, y medición de la superficie con daños.

* Permiten recoger información detallada de pies y focos afectados, en particular de características de estación, variables selvicolas y agentes bióticos y abióticos.

* Posibilidad realizar estudios de evolución temporal y espacial de los daños.

Sin embargo, el uso de censos presenta una serie de inconvenientes que debe ser tenidos encuentra, como son:

* Los procedimientos de inventario son caros, lo que limita considerablemente su repetición en periodos cortos de tiempo.

* La definición de áreas afectadas, y el reconocimiento en campo de las mismas. En muchas ocasiones es difícil hablar de focos individuales, y se observan grandes extensiones con daños de diferente nivel.

* La información se recoge fundamentalmente sobre pies con avanzado estado de daños, lo que dificulta los estudios de relación causa-efecto, siendo difícil discernir la evolución del proceso de afectación, así como los agentes asociados, al estar normalmente presentes varios de ellos.

En conjunto, los censos se consideran procedimientos de evaluación de daños adecuados para la estimación de la afectación de plagas y enfermedades forestales en un momento determinado, pero son difíciles de mantener en el tiempo, en particular en procesos que presentan patrones de variación espacio-temporal muy importante. 

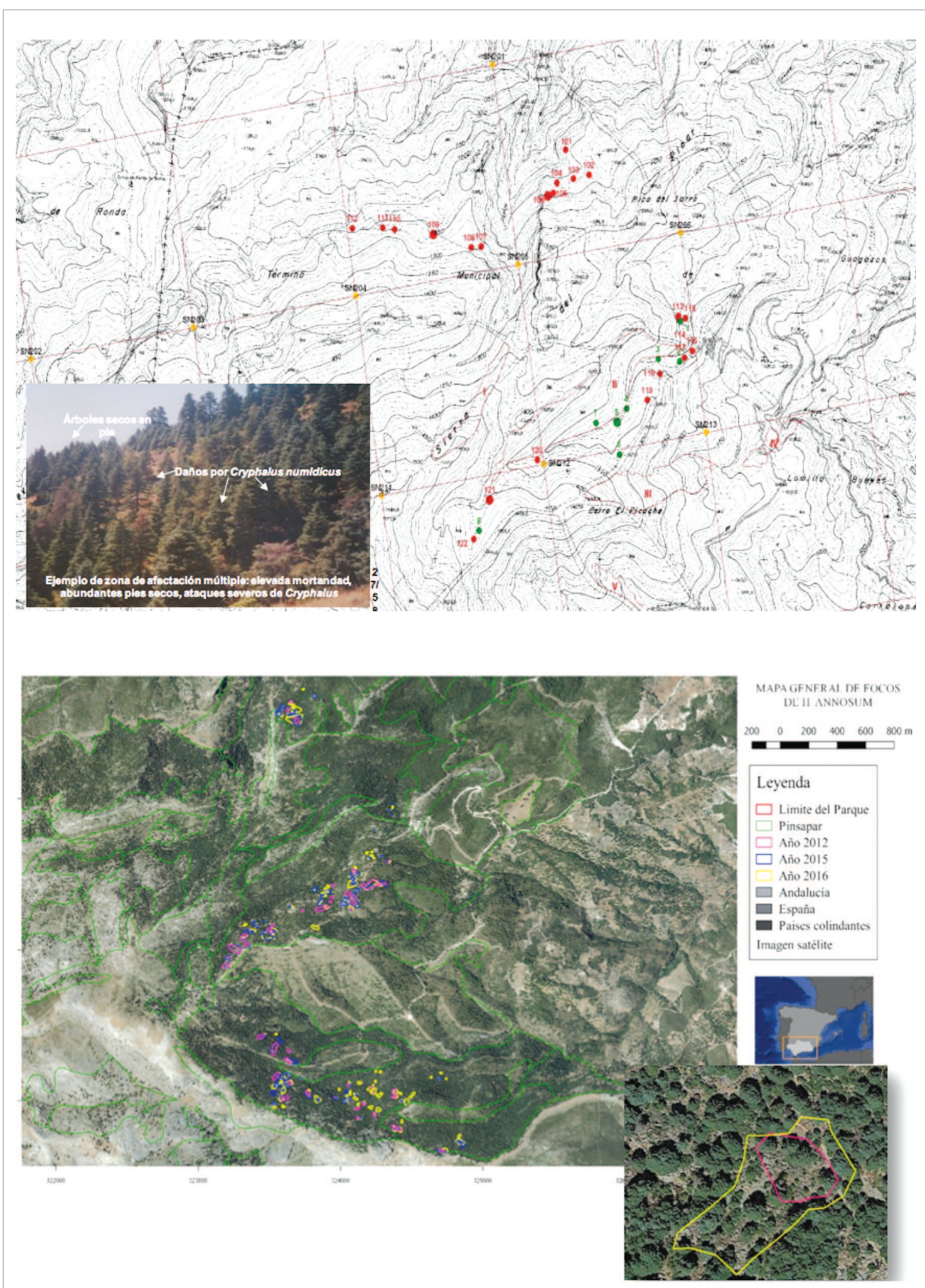

Figura 5. Cartografía de focos de Heterobasidion annosum en el Puerto del Saucillo (Sierra de las Nieves, superior) y actualización de focos mediante ortofotos del Plan Nacional de Teledetección (inferior). 


\subsection{Otras aplicaciones de la información procedente de censos}

\subsubsection{Uso de teledetección para la actualización de censos}

Uno de los aspectos interesantes de los censos, es que pueden integrar cartografía que, a su vez, se puede actualizar mediante teledetección (Figura 6), generando series temporales de un alto potencial interpretativo (Backsen y Howell, 2013; García Baena, 2018). En otras ocasiones los censos se utilizan para estudios de distribución de daños a partir de imágenes de satélite (Rullan-Silva et al., 2013; McDowell et al., 2015; Trujillo-Toro y Navarro-Cerrillo, 2019).

\subsubsection{Estudio de procesos de dinámica forestal}

La información procedente de censos se puede utilizar para estudiar la dinámica de la vegetación en masas afectadas por agentes bióticos. En concreto para el caso de los pinsapares andaluces se ha utilizado la información procedente de inventarios forestales realizados en áreas afectadas por $H$. annosum para estudiar la tendencia de sustitución de especies en el regenerado dentro de los focos(Navarro-Cerrillo et al., 2014).

\section{Parcelas monitorizadas}

El nivel de mayor detalle para conocer la incidencia de un agente biótico causante de plaga es a escala de parcela o árbol individual. Este diseño permite el control de múltiples factores, incrementando el poder estadístico de la información (Gent et al., 2017). Las parcelas monitorizadas permiten obtener variables del arbolado relacionadas con procesos fisiológicos, muy útiles sobre todo en procesos de interacción planta-patógeno que presentan síntomas inespecíficos o de difícil evaluación, como es el de los procesos de decaimiento de masas de encinar y alcornocal caso en Andalucía debido a la incidencia de "la seca" (Brasier, 1996; Navarro Cerrillo et al., 2001; Sánchez et al., 2002).

Las parcelas monitorizadas se pueden clasificar según su frecuencia de información sea discreta o contínua. Un ejemplo de parcelas monitorizadas con medidas discretas sería una red de parcelas de selvicultura, como la red de selvicultura adaptativa en masas repobladas de Pinus sylvestris L. y Pinus nigra J.F. Arnold de la Sierra de los Filabres. Esta red cuenta con 21 parcelas instaladas en 2010, con el objetivo de evaluar diferentes estrategias de manejo ante el cambio climático (Navarro-Cerrillo et al., 2019). La realización de visitas y toma de medidas de forma recurrente, las convierte en una potente fuente de información y de alerta ante la incidencia de enfermedades y plagas.

Las parcelas de medidas continuas son las más interesantes para el estudio de los procesos de interacción planta-patógeno. Las medidas continuas del estado fisiológico del arbolado, principalmente de los parámetros relacionados con la fotosíntesis, resulta de especial utilidad en parcelas afectadas por procesos de decaimiento o 


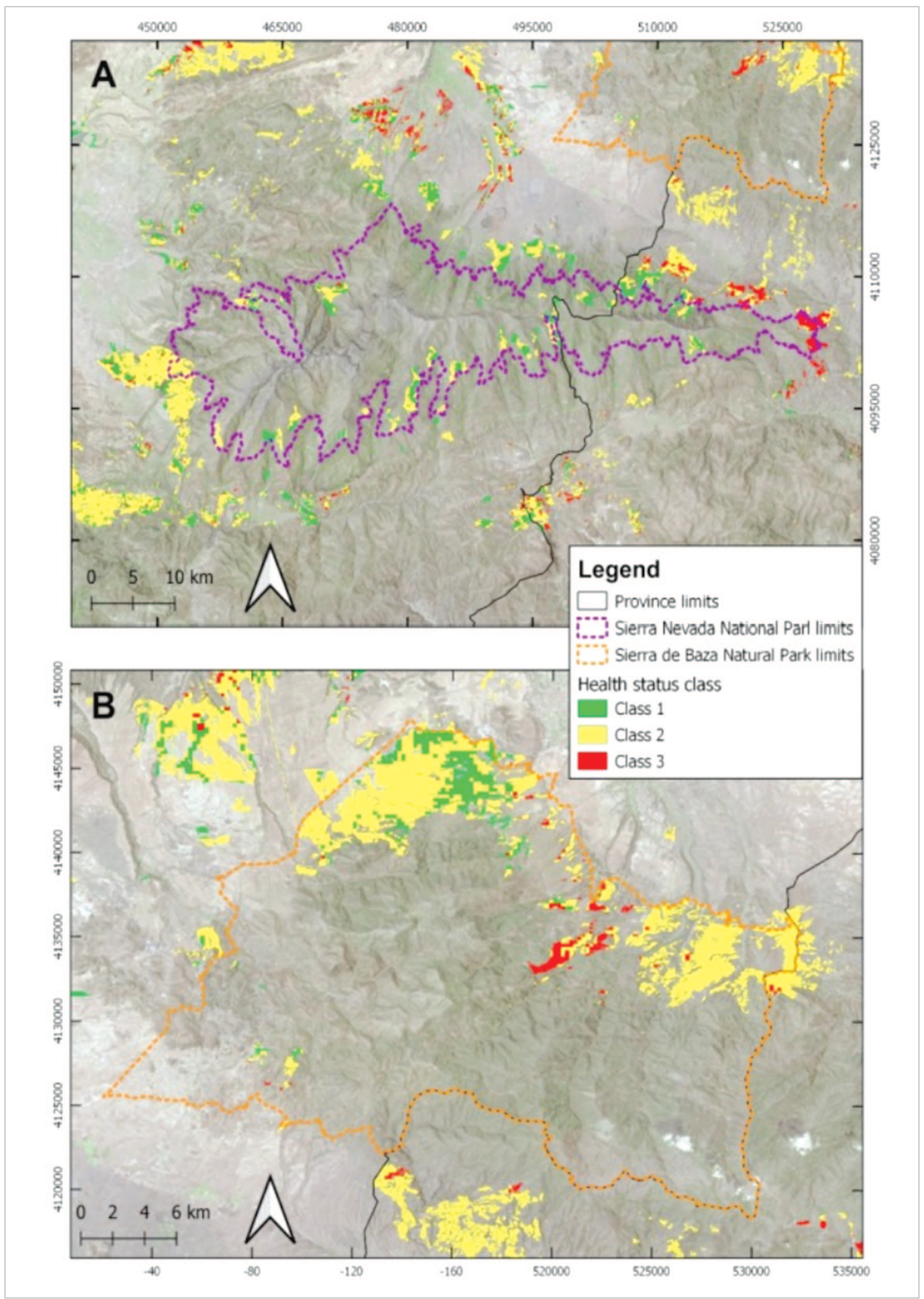

Figura 6. Cartografía de focos de daños de Candidatus Phytoplasmapini en Sierra de Baza-Sierra Nevada mediante series temporales de imágenes Landsat (Trujiillo-Toro y Navarro-Cerrillo, 2019). 
agentes de difícil cuantificación, como el caso de la podredumbre de raíz causada por Phytophthora cinnamomi (Maurel et al., 2004; Ruiz Gómez et al., 2018).

Para la obtención de variables ecofisiológicas en continuo se utilizan diferentes estrategias, desde la instalación de estructuras complejas y costosas, como las torres de Eddy covarianza, o detectores infrarrojos de intercambio gaseoso (IRGA's) instalados en continuo (Tognetti et al., 1998; Wilson et al., 2001), a otras medidas de estimadores indirectos de la fotosíntesis. Entre estos estimadores, el más extendido es la medida del flujo de savia (Köstner et al., 2017). Estas medidas han demostrado un gran potencial en la discriminación de diferencias en el comportamiento de especies mediterráneas sometidas a diferentes tipos e intensidades de estrés (Poyatos et al., 2016).

En este tipo de parcelas, cada vez es más frecuente incorporar redes inalámbricas de sensores. La nueva generación de sensores basados en tecnología IoT (Internet of Things) presenta importantes ventajas respecto a los sistemas de sensorización tradicionales (Burgess et al., 2010) (Figura 7). Estos sistemas suponen una importante reducción de coste frete a los sensores tradicionales. Los sensores inalámbricos aportan información muy precisa para detectar cambios con mayor rapidez que los enfoques tradicionales basados en medidas visuales, o incluso procedentes de teledetección. La evolución de estos sistemas posibilita el establecimiento de redes de parcelas cada vez más extensas que provean de información fisiológica detallada de diferentes ecosistemas forestales.

\subsection{Ventajas e inconvenientes de las parcelas monitorizadas:}

Las parcelas de monitorización de ecosistemas forestales presentan una serie de ventajas relacionadas con la evaluación de la incidencia de plagas y enfermedades forestales:

* Las parcelas monitorizadas permiten obtener una información de variables ecofisiológicas con un nivel de detalle muy superior al de cualquier otro tipo de sistemas de seguimiento.

* Permiten el establecimiento de diseños factoriales equilibrados, lo cual dota a la información de una potencia estadística que no es comparable a la de los datos de redes aleatorias o sistemáticas.

* El tamaño reducido de las parcelas permite un control exhaustivo de las condiciones ambientales.

* Tienen la escala ideal para estudiar los mecanismos de interacción entre planta y patógeno, reuniendo cierta capacidad de control experimental con el desarrollo de los procesos estudiados bajo condiciones ambientales reales. La información obtenida de este tipo de parcelas es más fácilmente escalable que la obtenida en ensayos de laboratorio o bajo condiciones controladas. 


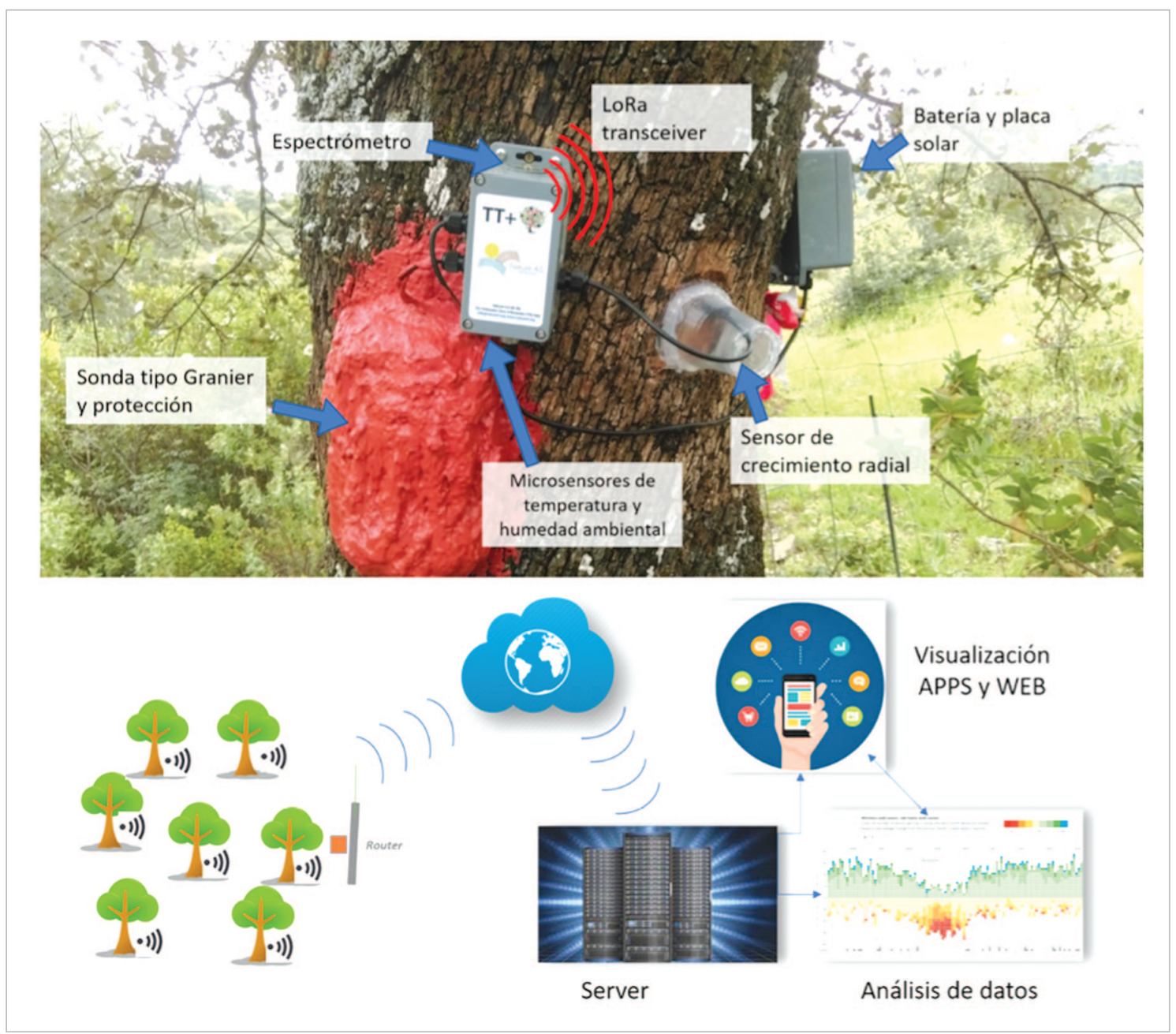

Figura 7. Medida de flujo de savia y monitorización de condiciones ambientales con sensores inalámbricos. Arriba: configuración de un sensor TreeTalker (Nature 4.0, Roveneto, Italia) en una parcela de encinar en la provincia de Córdoba (ERSAF-Universidad de Córdoba). Abajo, funcionamiento esquemático de una parcela con sensores inalámbricos y almacenamiento remoto de datos.

Sin embargo, las parcelas monitorizadas también presentan algunos inconvenientes que se deben de tener en cuenta para su correcta implementación:

* La monitorización de las parcelas en continuo es por norma general el método más costoso de obtención de datos en masas forestales, si bien esto está cambiando gracias a los nuevos sensores inalámbricos.

* Las parcelas sensorizadas requieren de un mantenimiento costoso en tiempo y recursos, además de una monitorización adecuada de su funcionamiento. Esto a la vez conlleva la necesidad de contar con personal altamente especializado y con conocimientos no sólo de los procesos estudiados, sino del funcionamiento del sistema y del manejo de grandes cantidades de datos. 
* Por último, el análisis de la información obtenida es más complejo, y requiere en muchas ocasiones de la colaboración de equipos multidisciplinares que aporten conocimientos especializados de áreas como la fisiología vegetal, la patología forestal, y la computación y análisis de datos.

\section{Conclusiones}

La gestión de los ecosistemas forestales en un entorno globalizado, dominado por las perturbaciones asociadas al cambio climático o a la incidencia de especies patógenas invasoras, es uno de los retos más importantes en la gestión forestal actual (Prospero y Cleary, 2017). En un contexto en el que la escasa rentabilidad económica de los sistemas forestales y la falta de valorización de los servicios ecosistémicos presentan una dificultad añadida en la getión de nuestros bosques, se hace más necesario que nunca el uso de herramientas de gestión eficaces y precisas. El análisis de datos procedentes de diferentes fuentes de información, apoyados por técnicas de teledetección y herramientas de modelización y computación estadística ofrecen alternativas robustas al inventario y al análisis clásico de las masas forestales.

En Andalucía, la presencia de una red de equilibrios biológicos en bosques (Red SEDA), establecida siguiendo estandares europeos ha permitido disponer de una estructura espacial y técnica óptima para el seguimiento de plagas y enfermedades forestales, como así se ha demostrado tanto desde el punto de vista de la gestión como de la investigación. Por otra parte, las sinergias entre esta red y otros sistemas de evaluación del estado de los bosques (inventarios, censos y parcelas), que proveen de datos con mayor escala de detalle, permiten crear sistemas complementarios de inspección, seguimiento y control para garantizar la bioseguridad forestal en Andalucía. Por último, estimamos necesario incorporar otras aproximaciones metodológicas, como la teledetección, la sensorización y la ciencia ciudadana, que refuercen y mejoren las herramientas actuales de monitorización y estudio de las enfermedades y los procesos de decaimiento en ecosistemas forestales.

\section{Agradecimientos:}

Los autores quieren agradecer al Departamento de Sanidad Forestal de la Consejería de Agricultura, Pesca y Desarrollo Rural de la Junta de Andalucía, y a la Agencia de Medio Ambiente y Agua la colaboración prestada, representados por Ángel Carrasco Gotarredona, Sixto Rodríguez y José M Ruiz Navarro, y a todos los técnicos de la Red SEDA por su colaboración permanente con los trabajos desarrollados por el grupo de investigación ERSAF. también agradecemos el apoyo económico de los diferentes convenios establecidos entre la Junta de Andalucía y ERSAF, así como los proyectos ESPECTRAMED (CGL2017-86161-R), Horizon 2020 ECOPOTENTIAL (No.641762), ISO-Pine (UCO-1265298) y Red SilvAdapt (RED2018102719-T). Los autores agradecen también la financiación del "Plan Propio de Investigación de la Universidad de Córdoba, 2019”. 


\section{Bibliografía}

Ayres, M.P.; Lombardero, M.J.; 2017. Forest pests and their management in the Anthropocene. Canadian Journal of Forest Research 48, 292-301. https://doi.org/10.1139/cjfr-2017-0033

Backsen, J.C.; Howell, B.; 2013. Comparing aerial detection and photo interpretation for conducting forest health surveys. Western Journal of Applied Forestry 28, 3-8. https:// doi.org/10.5849/wjaf.12-010

Boyd, I.L.; Freer-Smith, P.H.; Gilligan, C.A.; Godfray, H.C.J.; 2013. The consequence of tree pests and diseases for ecosystem services. Science [Internet] 342. Available from: https://science.sciencemag.org/content/342/6160/1235773. https://doi.org/10.1051/fo rest: 19960217

Brasier, C.M.; 1996. Phytophthora cinnamomi and oak decline in southern Europe. Environmental constraints including climate change. Annales des Sciences Forestières 53, 347-358. https://doi.org/10.1051/forest:19960217

Burgess, S.S.O.; Kranz, M.L.; Turner, N.E.; Cardell-Oliver, R.; Dawson, T.E.; 2010. Harnessing wireless sensor technologies to advance forest ecology and agricultural research. Agricultural and Forest Meteorology 150, 30-37. https://doi.org/10.1016/j.agrformet.2009.08.002

Cobos Suárez, P.; 1994. Estado fitosanitario de los pinsapares andaluces. En: Ruiz de la Torre, J.; García Viñas, J.; Oria de Rueda Salguero, J.; Cobos Suárez, J.M.; López Quintanilla, J.; Alvarez Calvente, M.; Arista, M.; Talavera, S.; Herrera, J.; editores: Gestión y conservación de los pinsapares andaluces Asociación Forestal Andaluza.

Duque-Lazo, J.; van Gils, H.; Groen, T.A.; Navarro-Cerrillo, R.M.; 2016. Transferability of species distribution models: The case of Phytophthora cinnamomi in Southwest Spain and Southwest Australia. Ecological Modelling 320, 62-70. https://doi.org/10. 1016/j.ecolmodel.2015.09.019

Duque-Lazo, J.; Navarro-Cerrillo, R.M.; 2017. What to save, the host or the pest? The spatial distribution of xylophage insects within the Mediterranean oak woodlands of Southwestern Spain. Forest Ecology and Management 392, 90-104. https://doi.org/ 10.1016/j.foreco.2017.02.047

Duque-Lazo, J.; Navarro-Cerrillo, R.M.; van Gils, H.; Groen, T.A.; 2018. Forecasting oak decline caused by Phytophthora cinnamomi in Andalusia: Identification of priority areas for intervention. Forest Ecology and Management 417, 122-136. https://doi.org/ 10.1016/j.foreco.2018.02.045

García Baena J., 2018. Cartografía de focos de Heterobasidion annosum (Fr.) Bref en el Parque Nacional de Sierra de las Nieves: análisis de causalidad (Tesina Fin de Master).

Gent, D.H.; Esker, P.D.; Kriss, A.B.; 2017. Statistical power in plant pathology research. Phytopathology 108, 15-22. https://doi.org/10.1094/PHYTO-03-17-0098-LE

Köstner, B.; Falge, E.; Alsheimer, M.; 2017. Sap flow measurements. En: Foken T, editor. Energy and Matter Fluxes of a Spruce Forest Ecosystem Ecological Studies Cham: Springer International Publishing. p 99-112. Available from: https://doi.org/10.1007/ 978-3-319-493893_5

Leather, S.; 2005. Insect sampling in forest ecosystems. Blackwell Science Ltd. https:// doi.org/10.1002/9780470750513

Loehle, C.; Idso, C.; Bently Wigley, T.; 2016. Physiological and ecological factors influencing recent trends in United States forest health responses to climate change. Forest Ecology and Management 363, 179-189. https://doi.org/10.1016/j.foreco.2015.12. 042 
López Quintanilla, J.; 2013. Los pinsapares en Andalucia (Abies pinsapo Boiss.): conservación y sostenibilidad en el siglo XXI. Servicio de Publicaciones. Available from: https://dialnet.unirioja.es/servlet/libro? codigo $=545036$

Lorenz, M.; 1995. International co-operative programme on assessment and monitoring of air pollution effects on Forests-ICP Forests-. Water, Air, \& Soil Pollution 85, 1221-1226. https://doi.org/10.1007/BF00477148

Maurel, M.; Robin, C.; Simonneau, T.; Loustau, D.; Dreyer, E.; Desprez-Loustau, M.L.; 2004. Stomatal conductance and root-to-shoot signalling in chestnut saplings exposed to Phytophthora cinnamomi or partial soil drying. Functional Plant Biology 31, 41-51. https://doi.org/10.1071/FP03133

McDowell, N.G.; Coops, N.C.; Beck, P.S.A.; Chambers, J.Q.; Gangodagamage, C.; Hicke, J.A.; Huang, C.; Kennedy, R.; Krofcheck, D.J.; et al., 2015. Global satellite monitoring of climate-induced vegetation disturbances. Trends in Plant Science 20, 114-123. https://doi.org/10.1016/j.tplants.2014.10.008

Muñoz López, C.; 2007. Sanidad forestal: guía en imágenes de plagas, enfermedades y otros agentes presentes en los bosques. Mundi-Prensa Libros.

Navarro Cerrillo, R.M.; Fernández Rebollo, P.; Ruiz Navarro, J.; Vidiella Salaberry, A.; 2001. El síndrome de la seca en masas de Quercus spp. En Andalucía. Congreso Forestal Español [Internet]. Available from: http://secforestales.org/publicaciones/in dex.php/congresos_forestales/article/view/15866

Navarro Cerrillo, R.M.; López Quintanilla, J.; Calzado Martínez, C.; Trapero Casas, A.; Sánchez Hernández, E.; 2003. Censo de focos de Heterobasidion annosum (Fr.) Bref. en ecosistemas de pinsapo. Boletín de sanidad vegetal. Plagas 29 (4), 581-592, 2003) [Internet]. Available from: http://helvia.uco.es/xmlui/handle/10396/2431

Navarro-Cerrillo, R.; Calzado Martínez, C.; 2001. Manual de campo para establecimiento de los puntos de la Red de Equilibrios Biológicos en Ecosistemas con presencia de pinsapo (Abies pinsapo Boiss.). Servicio de Ordenación de los Recursos Forestales. Consejería de Medio Ambiente. Junta de Andalucía.

Navarro-Cerrillo, R.; Camarero, J.; Manzanedo, R.; Sánchez-Cuesta, R.; Lopez Quintanilla, J.; Sánchez Salguero, R.; 2014. Regeneration of Abies pinsapo within gaps created by Heterobasidion annosum-induced tree mortality in southern Spain. iForest - Biogeosciences and Forestry 7, 209-215. https://doi.org/10.3832/ifor0961-007

Navarro-Cerrillo, R.; Duque-Lazo, J.; Manzanedo, R.; Sánchez-Salguero, R.; Palacios-Rodriguez, G.; 2018. Climate change may threaten the southernmost Pinus nigra subsp. salzmannii (Dunal) Franco populations: an ensemble niche-based approach. iForest - Biogeosciences and Forestry 11, 396-405. https://doi.org/10.3832/ifor2588-011

Navarro-Cerrillo, R.; Fernández Rebollo, P.; Ruiz Navarro, J.M.; 2000. Manual de campo para establecimiento de los puntos de la Red Andaluza de Daños sobre ecosistemas forestales en Andalucía. Servicio de Ordenación de los Recursos Forestales. Consejería de Medio Ambiente. Junta de Andalucía.

Navarro-Cerrillo, R.M.; Sánchez-Salguero, R.; Rodriguez, C.; Duque Lazo, J.; Moreno-Rojas, J.M.; Palacios-Rodriguez, G.; Camarero, J.J.; 2019. Is thinning an alternative when trees could die in response to drought? The case of planted Pinus nigra and P. Sylvestris stands in southern Spain. Forest Ecology and Management 433, 313-324. https://doi.org/10.1016/j.foreco.2018.11.006

Poyatos, R.; Granda, V; Molowny-Horas, R.; Mencuccini, M.; Steppe, K.; Martínez-Vilalta, J.; 
2016. SAPFLUXNET: towards a global database of sap flow measurements. Tree Physiology 36, 1449-1455. https://doi.org/10.1093/treephys/tpw110

Prospero, S.; Cleary, M.; 2017. Effects of host variability on the spread of invasive forest diseases. Forests 8, 80. https://doi.org/10.3390/f8030080

Rodriguez-Vallejo, C.; Navarro-Cerrillo, R.M.; 2019. Contrasting response to drought and climate of planted and natural Pinus pinaster Aiton forests in Southern Spain. Forests 10, 603. https://doi.org/10.3390/f10070603

Ruiz Gómez, F.J.; Pérez-de-Luque, A.; Sánchez-Cuesta, R.; Quero, J.; Navarro Cerrillo, R.; 2018. Differences in the response to acute drought and Phytophthora cinnamomi Rands Infection in Quercus ilex L. seedlings. Forests 9, 634. https://doi.org/10.3390/f9100634

Ruiz Gómez, F.J.; Navarro-Cerrillo, R.M.; Pérez-de-Luque, A.; Oßwald, W.; Vannini, A.; Morales-Rodríguez, C.; 2019. Assessment of functional and structural changes of soil fungal and oomycete communities in holm oak declined dehesas through metabarcoding analysis. Scientific Reports 9, 5315. https://doi.org/10.1038/s41598-019-41804-y

Ruiz-Benito, P.; García-Valdés, R.; 2016. Inventarios forestales para el estudio de patrones y procesos en Ecología. Revista Ecosistemas 25, 1-5-5. https://doi.org/10.7818/EC OS.2016.253.01

Rullan-Silva, C.; Olthoff, A.; Delgado de la Mata, J.; Pajares Alonso, J.; 2013. Remote monitoring of forest insect defoliation. A review. Forest Systems 22, 377-391. https://doi. org/10.5424/fs/2013223-04417

Sánchez, M.; Caetano, P.; Ferraz, J.; Trapero, A.; 2002. Phytophthora disease of Quercus ilex in south-western Spain. Forest Pathology 32, 5-18. https://doi.org/10.1046/j.14 390329.2002.00261.x

Sánchez, M.E.; Luchi, N.; Jiménez, J.J.; Vita, P.D.; Sánchez, J.E.; Trapero, A.; Capretti, P.; 2007. An isolated population of Heterobasidion abietinum on Abies pinsapo in Spain. Forest Pathology 37, 348-356. https://doi.org/10.1111/j.1439-0329.2007.00514.x

Sánchez Peña, G.; Torres Martínez, B.; Prieto González, M.; 2009. Evolución de la vitalidad de los bosques españoles y en Europa: 1987-2008., más de 20 años del seguimiento de los bosques en Europa. Congreso Forestal Español [Internet]. Available from: http://secforestales.org/publicaciones/index.php/congresos_forestales/article/view/17124

Sánchez-Salguero, R.; Camarero, J.J.; Dobbertin, M.; Fernández-Cancio, Á.; Vilà-Cabrera, A.; Manzanedo, R.D.; Zavala, M.A.; Navarro-Cerrillo, R.M.; 2013. Contrasting vulnerability and resilience to drought-induced decline of densely planted vs. natural rear-edge Pinus nigra forests. Forest Ecology and Management 310, 956-967. https:// doi.org/10.1016/j.foreco.2013.09.050

Sinclair, W.; Lyon, H.; 2005. Diseases of trees and shrubs. Edición: 2 ed. Ithaca: Cornell University Press.

Smith, I.; Dunez, J.; Lelliot, R.; Phillips, D.; Archer, S.; 1992. Manual de enfermedades de las plantas. Madrid: Mundi Prensa Libros S.A.

SSF - DGDRyPF., 2012. Red de Seguimiento a gran Escala de Daños en los Bosques (Red de Nivel I). Manual de Campo. Área de Inventario y Estadísticas Forestales de la Dirección General de Desarrollo Rural y Política Forestal. Ministerio de Agricultura., Alimentación y Medio Ambiente.

Tognetti, R.; Longobucco, A.; Miglietta, F.; Raschi, A.; 1998. Transpiration and stomatal behaviour of Quercus ilex plants during the summer in a Mediterranean carbon dioxide spring. Plant., Cell \& Environment 21., 613-622. https://doi.org/10.1046/j.1365-3040.1998.00301.x 
Trujillo-Toro, J.; Navarro-Cerrillo, R.M.; 2019. Analysis of Site-dependent Pinus halepensis Mill. defoliation caused by candidatus Phytoplasma pini through shape selection in Landsat time series. Remote Sensing 11., 1868. https://doi.org/10.3390/rs11161868

Trumbore, S.; Brando, P.; Hartmann, H.; 2015. Forest health and global change. Science 349., 814-818. https://doi.org/10.1126/science.aac6759

Wilson, K.B.; Hanson, P.J.; Mulholland, P.J.; Baldocchi, D.D.; Wullschleger, S.D.; 2001. A comparison of methods for determining forest evapotranspiration and its components: sap-flow, soil water budget, eddy covariance and catchment water balance. Agricultural and Forest Meteorology 106., 153-168. https://doi.org/10.1016/S0168-1923(00) 00199-4 
